\title{
Cyclic AMP Restores Appressorium Formation Inhibited by Polyamines in Magnaporthe grisea
}

\author{
Woo-Bong Choi, Shin-Ho Kang, Yin-Won Lee, and Yong-Hwan Lee
}

Department of Agricultural Biology and Research Center for New Bio-Materials in Agriculture, College of Agriculture and Life Sciences, Seoul National University, Suwon 441-744, Korea.

Current address of W.-B. Choi: Department of Plant Pathology and Physiology, Clemson University, Clemson, SC 29634.

Accepted for publication 8 October 1997.

\begin{abstract}
Choi, W.-B., Kang, S.-H., Lee, Y.-W., and Lee, Y.-H. 1998. Cyclic AMP restores appressorium formation inhibited by polyamines in Magnaporthe grisea. Phytopathology 88:58-62.

Magnaporthe grisea, the causal agent of rice blast, forms a domeshaped melanized infection structure, an appressorium, to infect its host. Environmental cues that induce appressorium formation in this fungus include the hydrophobicity and hardness of the contact surface and chemicals produced by the host. An elevated concentration of intracellular cyclic AMP (cAMP) has been implicated in appressorium differentiation in M. grisea. Polyamines (putrescine, spermidine, and spermine) are involved in cell growth and differentiation in a wide range

ferentiation in $M$. grisea, intracellular polyamines were quantified, and the effects of polyamines and polyamine biosynthesis inhibitors on conidial germination and appressorium formation were tested. High levels of polyamines were detected in freshly collected spores, but the levels decreased during conidial germination. Spermidine was found to be the major component. Polyamines and polyamine biosynthesis inhibitors did not affect conidial germination, but polyamines specifically impaired appressorium formation. Furthermore, exogenous addition of cAMP restored appressorium formation inhibited by polyamines. These results suggest that polyamines may reduce intracellular cAMP levels in $M$. grisea, leading to the inhibition of appressorium formation.
\end{abstract} of organisms. To understand the role of polyamines in appressorium dif-
Magnaporthe grisea (T. T. Hebert) Yaegashi \& Udagawa (anamorph: Pyricularia grisea (Cooke) Sacc.) is a typical hermaphroditic ascomycete and the causal agent of rice blast, the most destructive disease of rice throughout the rice-growing areas of the world. Controlling this disease has been dependent on breeding resistant cultivars and the application of chemical fungicides. However, the frequent appearance of new races has reduced the effectiveness of resistant cultivars in the field (18). Furthermore, environmental regulations have also restricted the use of chemical fungicides. An understanding of infection mechanisms is a prerequisite for the development of new strategies to control this disease.

M. grisea, like many other plant pathogens, forms a specialized infection structure, an appressorium, to adhere tightly to and then penetrate its host. The differentiation and maturation of appressoria are critical steps for successful infection. Appressorium formation in plant-pathogenic fungi is induced by environmental stimuli including thigmotropic and chemical signals from the host surface $(7,20)$. Environmental cues inducing appressorium formation in $M$. grisea include the hydrophobicity and hardness of the contact surface and chemicals from the plant surface $(5,15,32)$. Intracellular signaling systems involved in appressorium differentiation in this fungus have also been investigated during recent years. Lee and Dean (15) found that appressorium formation was induced on noninductive surfaces by the addition of exogenous cyclic AMP (cAMP). Twenty isolates of $M$. grisea originating from rice and other gramineous plants also formed appressoria on noninductive surfaces in the presence of cAMP (14). Furthermore,

Corresponding author: Y.-H. Lee; E-mail address: yonglee@plaza.snu.ac.kr

Publication no. P-1997-1114-01R

(C) 1998 The American Phytopathological Society targeted disruption of the catalytic subunit gene (cpkA) of cAMPdependent protein kinase in $M$. grisea abolished the ability to form normal appressoria (16). Recently, Beckerman and Ebbole (3) reported that $M P G 1$-disrupted mutants, which are unable to form appressoria, formed appressoria when cAMP was added. These experiments suggest that cAMP plays an important role in the regulation of appressorium formation in $M$. grisea.

Polyamines (putrescine, spermidine, and spermine) are ubiquitous organic cations of low molecular weight that bind to polyanionic macromolecules like DNA, RNA, and phospholipids in cells (8). Putrescine is synthesized from ornithine by ornithine decarboxylase (ODC) (EC 4.1.1.17) or from arginine by arginine decarboxylase (ADC) (EC 4.1.1.19) via agmatine and $N$-carbamoylputrescine. Polyamines are involved in cell growth and development in a wide range of organisms $(24,28)$. In fungi, polyamines have been reported to be important in cell differentiation during spore germination, dimorphic transition, and conidiation $(13,17,23,25)$. High levels of cellular polyamine lead to hyphal growth rather than yeastlike growth in the dimorphic fungus $\mathrm{Mu}$ cor racemosus (9). In Aspergillus flavus, a low putrescine/spermidine ratio is important for microcycle conidiation (13). Higher plants and bacteria utilize both the ornithine and the arginine pathways for the synthesis of putrescine, but most fungi are thought to have only the ODC pathway $(19,29)$. Therefore, ODC inhibitors and product analogs of polyamines have been tested for inhibition of mycelial growth and used to control plant diseases caused by fungi $(6,12,21,29)$. Recently, it was reported that polyamine biosynthesis inhibitors impair appressorium formation in Uromyces viciae-fabae (22). Furthermore, an inverse relationship between intracellular cAMP and polyamines has been reported in bacteria and fungi $(17,31)$. However, to date no attempts have been made to examine the effects of polyamines and polyamine biosynthesis inhibitors on conidial germination, mycelial growth, and appressorium formation in $M$. grisea. 
We demonstrate here that polyamines, including putrescine, spermidine, and spermine, specifically inhibit appressorium formation in $M$. grisea. Furthermore, the inhibition of appressorium formation by polyamines is overcome by the addition of exogenous cAMP.

\section{MATERIALS AND METHODS}

Fungal strain and cultural condition. M. grisea MG07 was used throughout the experiments and was routinely maintained on complete medium. Fungal cultures were grown on oatmeal agar (50 g of oatmeal per liter) at $21^{\circ} \mathrm{C}$ under fluorescent light to promote conidiation. Conidia were collected from 10-day-old cultures and washed twice with distilled water.

Conidial germination, mycelial growth, and appressorium formation. Conidial germination and appressorium formation were measured on GelBond film (FMC BioProducts, Rockland, Maine) as previously described (15). Briefly, a 50- $\mu$ d drop of a conidial suspension $\left(10^{4}\right.$ conidia per milliliter $)$ was placed on GelBond film, sealed in a moistened box, and incubated at room temperature for $20 \mathrm{~h}$, unless otherwise indicated. The percentages of germinated and germinating conidia induced to form appressoria were determined from direct microscopic examination of at least 100 conidia per replicate in at least four experiments, with three replicates per treatment. Mycelial disks (5 $\mathrm{mm}$ in diameter) from actively growing margins of colonies of $M$. grisea MG07 on complete medium were transferred to complete medium containing chemicals and incubated at $24^{\circ} \mathrm{C}$ for 5 days. Each experiment, with three replicates, was repeated at least twice. Putrescine, spermidine, spermine, methylglyoxal bis-(guanylhydrazone) (MGBG), $\mathrm{N}^{6}$-monobutyryl cAMP, and 3-isobutyl-1-methylxanthine (IBMX) were purchased from Sigma (St. Louis, Mo.). Difluoromethylornithine (DFMO) was kindly provided by C. K. Seoung (Chungnam National University, Taejon, Korea).

Extraction and analysis of polyamines. Potato-dextrose broth was inoculated with a conidial suspension and incubated at $25^{\circ} \mathrm{C}$ for $1,2,4,8$, and $24 \mathrm{~h}$. Germinating conidia were harvested by filtration, washed with distilled $\mathrm{H}_{2} \mathrm{O}$, and ground in ice-cold $5 \%$ perchloric acid. The homogenates were placed in an ice bath for $1 \mathrm{~h}$ and centrifuged at $26,000 \times g$ for $15 \mathrm{~min}$. The supernatant (perchloric acid-soluble) fraction was used for analysis of free polyamines (4). A 500- $\mu$ l portion of the sample extract was added to $1 \mathrm{ml}$ of $2 \mathrm{~N} \mathrm{NaOH}$, mixed with $10 \mu \mathrm{l}$ of benzoyl chloride, and vortexed for $10 \mathrm{~s}$. Then $2 \mathrm{ml}$ of water saturated with $\mathrm{NaCl}$ was added. Benzoylated polyamines were extracted in $2 \mathrm{ml}$ of diethyl ether and centrifuged at $5,000 \times g$ for $10 \mathrm{~min}$. A $1-\mathrm{ml}$ portion of the ether fraction was evaporated to dryness and dissolved in $100 \mu \mathrm{l}$ of methanol. Polyamines were analyzed with a Shimadzu LC-6A high-performance liquid chromatograph; a Zorbax octadecylsilane column $(4.6 \mathrm{~mm} \times 15 \mathrm{~cm}$; particle size $5 \mu \mathrm{m})$ (Dupont Co., Kyoto, Japan) with a mobile phase of $60 \%$ aqueous methanol, a flow rate of $1 \mathrm{ml} / \mathrm{min}$, and a detection wavelength of 254 $\mathrm{nm}$ was used. The calculation of the polyamine concentration in the extracts was based on the average area counts of each of the three standard polyamines.

\section{RESULTS}

Effect of polyamines and polyamine biosynthesis inhibitors on conidial germination, appressorium formation, and mycelial growth. Appressorium formation in M. grisea was inhibited by 1 to $5 \mathrm{mM}$ putrescine, spermidine, and spermine in a dosedependent manner (Table 1). Spermine was the most potent inhibitor, followed by putrescine and spermidine. However, appressorium formation was not affected by the polyamine biosynthesis inhibitors DFMO and MGBG. Appressoria formed in the presence of these inhibitors were well melanized and indistinguishable

TABLE 1. Effect of putrescine, spermidine, spermine, difluromethylornithine (DFMO), and methylglyoxal bis-(guanylhydrazone) (MGBG) on conidial germination and appressorium formation in Magnaporthe grisea $^{\text {a }}$

\begin{tabular}{lccc}
\hline Treatment & $\begin{array}{c}\text { Concentration } \\
(\mathrm{mM})\end{array}$ & $\begin{array}{c}\text { Conidial } \\
\text { germination } \\
(\% \pm \mathrm{SD})\end{array}$ & $\begin{array}{c}\text { Appressorium } \\
\text { formation } \\
(\% \pm \mathrm{SD})\end{array}$ \\
\hline Putrescine & 5 & $88.7 \pm 2.5$ & $1.7 \pm 1.5$ \\
Spermidine & 1 & $92.3 \pm 2.5$ & $17.0 \pm 5.3$ \\
& 5 & $90.7 \pm 2.1$ & $5.0 \pm 1.0$ \\
Spermine & 1 & $92.3 \pm 4.0$ & $79.3 \pm 4.5$ \\
& 5 & $91.3 \pm 3.1$ & $1.7 \pm 1.5$ \\
DFMO & 1 & $94.7 \pm 1.5$ & $8.0 \pm 2.6$ \\
& 5 & $94.3 \pm 1.2$ & $94.3 \pm 1.5$ \\
MGBG & 1 & $92.7 \pm 3.2$ & $93.7 \pm 2.1$ \\
& 5 & $96.0 \pm 1.5$ & $84.3 \pm 5.7$ \\
Untreated & 1 & $95.5 \pm 1.0$ & $91.3 \pm 5.5$ \\
\hline
\end{tabular}

a A conidial suspension ( $10^{4}$ conidia per milliliter) containing polyamine, DFMO, or MGBG was placed on the hydrophobic side of GelBond film and incubated in a moistened box at $25^{\circ} \mathrm{C}$. Appressorium formation was determined as described in Materials and Methods.

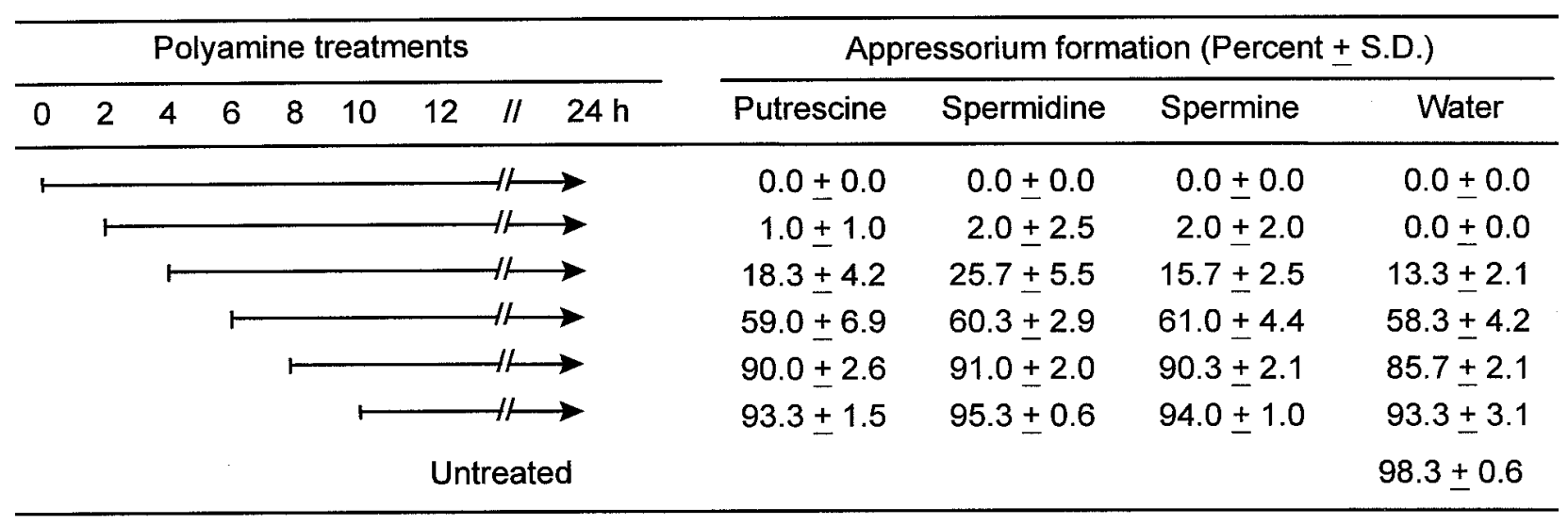

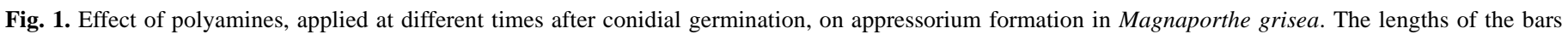

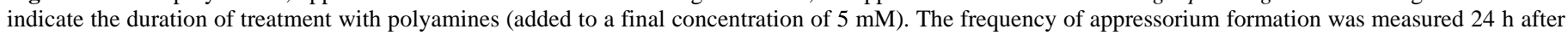

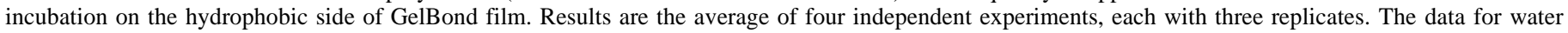
indicate the number of appressoria that had formed at the time of polyamine addition. 
from those formed in the untreated control. To investigate the inhibition window of polyamines on appressorium formation, putrescine, spermidine, and spermine were added to conidial suspensions on a hydrophobic surface at different times. Appressorium formation was completely blocked by the addition of a polyamine at the start of incubation or $2 \mathrm{~h}$ after the start of incubation. The number of appressoria formed in cultures to which a polyamine was added $4,6,8$, or $10 \mathrm{~h}$ after the start of incubation was similar to the number present at the time the chemical was added (Fig. 1). In other words, no additional appressoria developed after the polyamines were added. Conidial germination of $M$. grisea was not affected by treatment with polyamines at concentrations up to $5 \mathrm{mM}$ (Table 1). The three polyamines did not affect mycelial growth at $0.1 \mathrm{mM}$, but mycelial growth was significantly reduced at $10 \mathrm{mM}$ (data not shown). Spermine was the most effective inhibitor of mycelial growth of $M$. grisea, followed by spermidine and putrescine. MGBG strongly inhibited mycelial growth at $10 \mathrm{mM}$.

Effect of cAMP on inhibition of appressorium formation by polyamines. The inhibition of appressorium formation by putrescine, spermidine, or spermine on the hydrophobic surface of GelBond film was reversed by treatments with $10 \mathrm{mM} \mathrm{N} 6$-monobutyryl cAMP or $2.5 \mathrm{mM}$ IBMX (Table 2). $\mathrm{N}^{6}$-monobutyryl cAMP was much more effective than IBMX. IBMX did not restore appressorium formation inhibited by spermidine or spermine, although $45 \%$ of germlings formed appressoria in the presence of putrescine and IBMX. Appressoria induced by $\mathrm{N}^{6}$-monobutyryl cAMP in the presence of polyamines were well melanized and indistinguishable from those formed in the untreated control (Fig. 2). However, appressoria induced by IBMX in the presence of polyamine were small and irregularly shaped (data not shown).

1,16-Hexadecanediol also induces appressorium formation in $M$. grisea on a noninductive surface (5). This chemical also restored appressorium formation in $M$. grisea to a high level after it had been inhibited by polyamines (data not shown).

Polyamine concentration during conidial germination. Freshly collected conidia of $M$. grisea contained a high level of polyamines. The level of polyamines in the fungal cells decreased during conidial germination (Fig. 3). Spermidine was the major polyamine component in $M$. grisea, followed by spermine. Only trace amounts of putrescine were detected. Levels of spermidine decreased during conidial germination, whereas those of putrescine and spermine remained unchanged.

TABLE 2. Effect of $\mathrm{N}^{6}$-monobutyryl cyclic AMP (mb cAMP) and 3-isobutyl-1-methylxanthine (IBMX) on the inhibition of appressorium formation in Magnaporthe grisea by polyamines on a hydrophobic surface ${ }^{\mathrm{a}}$

\begin{tabular}{lc}
\hline Treatment & $\begin{array}{c}\text { Appressorium formation } \\
(\% \pm \mathrm{SD})\end{array}$ \\
\hline Putrescine & $1.7 \pm 1.5$ \\
Putrescine + mb cAMP & $86.0 \pm 2.6$ \\
Putrescine + IBMX & $44.7 \pm 4.0$ \\
& \\
Spermidine & $5.0 \pm 1.0$ \\
Spermidine + mb cAMP & $87.3 \pm 1.5$ \\
Spermidine + IBMX & $10.3 \pm 4.5$ \\
Spermine & $1.7 \pm 1.5$ \\
Spermine + mb cAMP & $91.0 \pm 2.6$ \\
Spermine + IBMX & $2.3 \pm 2.5$ \\
Untreated & $88.7 \pm 5.5$ \\
\hline
\end{tabular}

${ }^{a}$ A conidial suspension $\left(10^{4}\right.$ conidia per milliliter) containing polyamine, polyamine plus mb cAMP, or polyamine plus IBMX was placed on the hydrophobic side of GelBond film and incubated in a moistened box at $25^{\circ} \mathrm{C}$. Appressorium formation was determined as described in Materials and Methods. Polyamines, mb cAMP, and IBMX were added to final concentrations of 5,10 , and $2.5 \mathrm{mM}$, respectively.

\section{DISCUSSION}

In these experiments, we show that polyamines, including putrescine, spermidine, and spermine, specifically inhibit appressorium formation in $M$. grisea. Significantly, conidial germination is not affected by these polyamines or by the polyamine synthesis inhibitors DFMO and MGBG, at concentrations of $5 \mathrm{mM}$. These inhibitors did not affect appressorium formation. In contrast, appressorium formation in Uromyces viciae-fabae is inhibited by DFMO, while conidial germination remains unaffected (22). In M. grisea, the lack of an observable effect of DFMO suggests that putrescine and the other polyamines are not required for conidial germination and appressorium formation or that $M$. grisea contains the ADC pathway. Most fungi contain only the ODC pathway, but ADC activity has been detected in Ceratocystis minor and Verticillium dahliae (11). An alternative explanation is that this concentration of DFMO is not effective in M. grisea. Several fungi, including Septoria tritici and Ustilago maydis, have been reported to be insensitive to DFMO $(27,29)$, whereas many others, such as Phytophthora infestans and Sclerotium rolfsii, are highly sensitive $(2,26,30)$.

Freshly collected conidia contained high levels of polyamines, which decreased during conidial germination. Our results differ somewhat from other observations. In Mucor racemosus, low

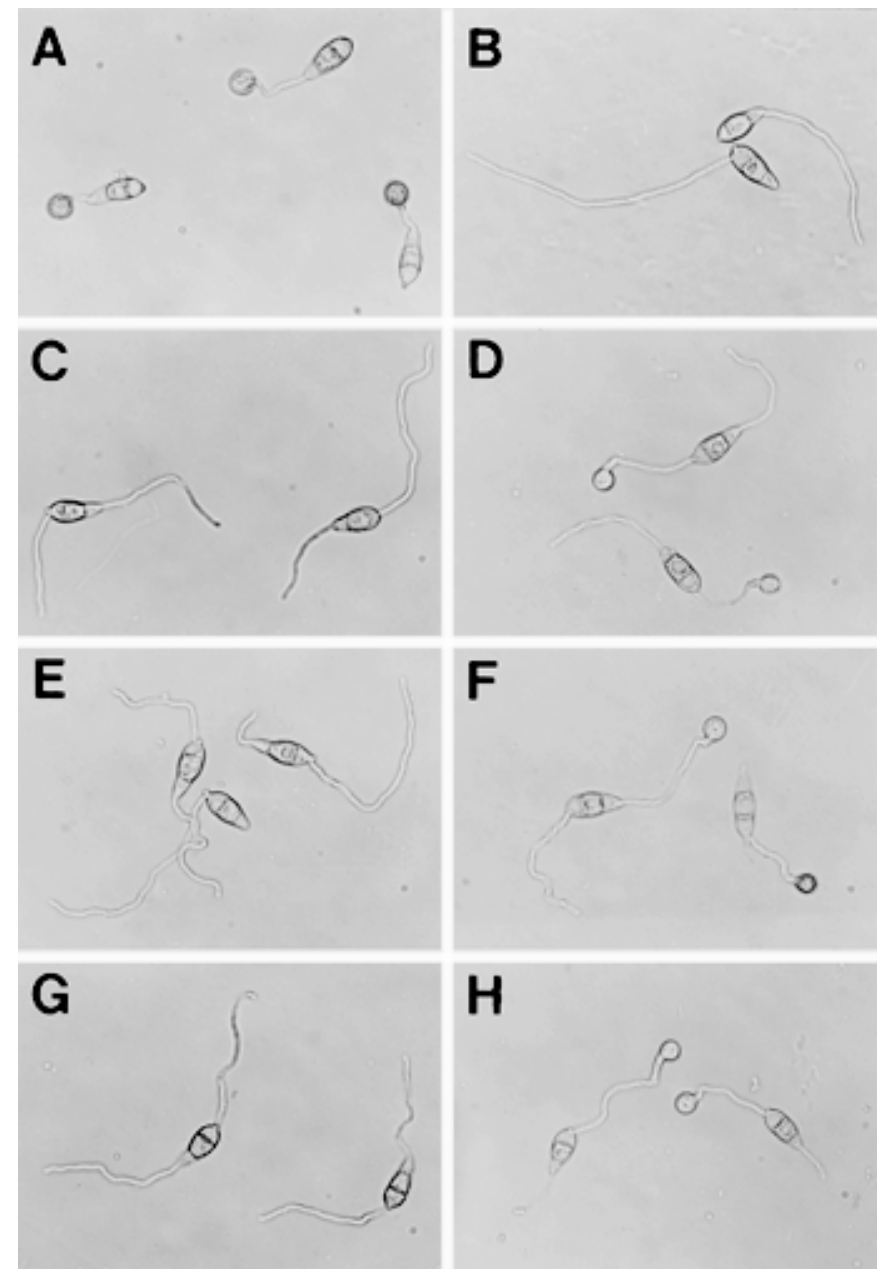

Fig. 2. Restoration of appressorium formation inhibited by polyamines in Magnaporthe grisea. A and B, Conidia germinated on the hydrophilic and hydrophobic sides of GelBond film, respectively. $\mathbf{C}, \mathbf{E}$, and $\mathbf{G}$, Inhibition of appressorium formation in the presence of putrescine, spermidine, and spermine, respectively, on the hydrophobic side of GelBond film. D, F, and $\mathbf{H}$, Appressorium formation restored by cyclic AMP after inhibition by putrescine, spermidine, and spermine, respectively, on the hydrophobic side of GelBond film. 
levels of polyamines were detected in spores, and high ODC activity was detected during early stages of germination (9). Spermidine was the most prevalent polyamine in $M$. grisea. Similar results have been observed in several other fungi, such as Phytophthora infestans and Pythium ultimum (30). However, in Aspergillus flavus putrescine was present at the highest concentration, followed by spermidine and spermine (13). During microcyclic spore differentiation in A. flavus, the level of putrescine dropped. However, the putrescine/spermidine ratio increased in $M$. grisea during conidial germination, because of a decrease in the level of spermidine.

Intracellular cAMP is an important modulator of appressorium formation in $M$. grisea $(15,16)$. The level of cAMP in the cells is regulated by two enzymes, adenylate cyclase and phosphodiesterase, for synthesis and degradation, respectively. $\mathrm{N}^{6}$-monobutyryl cAMP restored appressorium formation inhibited by polyamines at high frequencies (Table 2 and Fig. 2). This indicates that polyamine inhibition of appressorium formation in this fungus is upstream of where cAMP functions and suggests that polyamines may decrease intracellular cAMP levels. Because IBMX was unable to restore appressorium formation inhibited by polyamines, the inhibition of phosphodiesterase activity may not be sufficient to allow the accumulation of effective levels of cAMP in the presence of polyamines. Therefore, polyamine inhibition of appressorium formation may be due to the suppression of adenylate cyclase. It has been documented that polyamines depress cAMP synthesis by inhibiting adenylate cyclase in other organisms $(1,17)$. Also, ODC activity has been shown to be negatively regulated by cAMP in both eukaryotic and prokaryotic cells $(10,31)$. Alternatively, polyamines may directly or indirectly increase the requirement for cAMP by causing other cellular effects. In this case, the normal activity of adenylate cyclase might be insufficient to induce appressorium formation. The mechanism by which polyamines mediate cAMP metabolism in $M$. grisea remains to be elucidated.

Although the precise mechanisms involved in the inhibition of appressorium formation by polyamines are not clear, our data suggest that polyamines extent these effects through cAMP in $M$. grisea. Understanding the specific inhibition of appressorium differentiation is not only of interest in cell biology but also important in providing direction for the development of novel strategies to control rice blast disease.

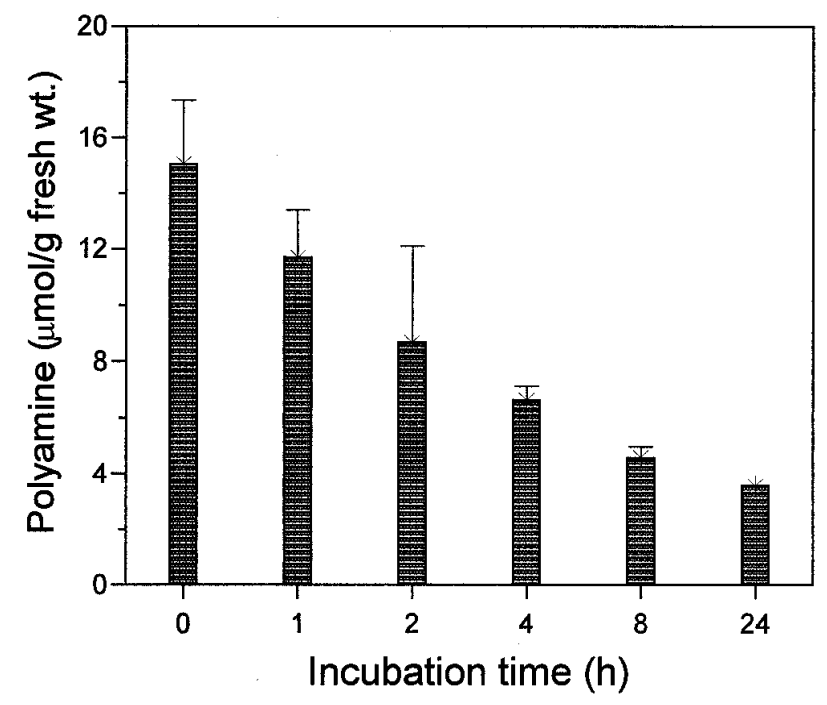

Fig. 3. Polyamine analysis by high-performance liquid chromatography during germination of conidia of Magnaporthe grisea. Polyamines were extracted from freshly collected conidia and germinating conidia 1, 2, 4, 8, and $24 \mathrm{~h}$ after inoculation of potato-dextrose broth. The polyamines include putrescine, spermidine, and spermine.

\section{ACKNOWLEDGMENTS}

This work was supported in part by the Korea Science and Engineering Foundation through the Research Center for New Bio-Materials in Agriculture at Seoul National University and the Korea-U.S. Cooperative Science Program.

\section{LITERATURE CITED}

1. Atmar, V. J., Westland, J. A., Garcia, G., and Kuehn, G. D. 1976. Adenylate cyclases Physarum polycephalum: Inhibition of a nuclear enzyme by polyamines. Biochem. Biophys. Res. Commun. 68:561-568.

2. Barker, J. H., Smith, T. A., Owen, W. H. 1993. Inhibition of polyamine metabolism in Phytophthora species. Mycol. Res. 97:1358-1362.

3. Beckerman, J. L., and Ebbole, D. J. 1996. MPG1, a gene encoding a fungal hydrophobin of Magnaporthe grisea, is involved in surface recognition. Mol. Plant-Microbe Interact. 9:450-456.

4. Flores, H. E., and Galstone, A. W. 1982. Analysis of polyamines in higher plants by high performance liquid chromatography. Plant Physiol. 69:701-706.

5. Gilbert, R. D., Johnson, A. M., and Dean, R. A. 1996. Chemical signals responsible for appressorium formation in the rice blast fungus Magnaporthe grisea. Physiol. Mol. Plant Pathol. 48:335-346.

6. Havis, N. D., Walters, D. R., Martin, W. P., Cook, F. M., and Robins, D. J. 1994. Fungicidal activity of three putrescine analogues. Pestic. Sci. 41: 71-76.

7. Hoch, H. C., Staples, R. C., Whitehead, B., Comeau, J., and Wolf, E. D. 1987. Signaling for growth orientation and cell differentiation by surface topography in Uromyces. Science 235:1659-1662.

8. Igarashi, K., Sakamoto, I., Goto, N., Kashiwagi, K., Homma, R., and Hirose, S. 1982. Interaction between polyamines and nucleic acids or phospholipids. Arch. Biochem. Biophys. 219:438-443.

9. Inderlied, C. B., Cihlar, R. L., and Sypherd, P. S. 1980. Regulation of ornithine decarboxylase during morphogenesis of Mucor racemosus. J. Bacteriol. 141:699-706.

10. Insel, P. A., and Fenno, J. 1978. Cyclic AMP-dependent protein kinase mediates a cyclic AMP-stimulated decrease in ornithine and $S$-adenosylmethionine decarboxylase activities. Proc. Natl. Acad. Sci. USA 75:862865.

11. Khan, A. J., and Minocha, S. C. 1989. Biosynthetic arginine decarboxylase in some phytopathogenic fungi. Life Sci. 44:1215-1222.

12. Khan, A. J., and Minocha, S. C. 1989. Polyamine biosynthetic enzymes and the effect of their inhibition on the growth of some phytopathogenic fungi. Plant Cell Physiol. 30:655-663.

13. Khurana, N., Saxena, R. K., Gupta, R., and Rajam, M. V. 1996. Polyamines as modulators of microcycle conidiation in Aspergillus flavus. Microbiology 142:517-523.

14. Lee, Y. H., and Choi, W. B. 1994. Uniformity among Magnaporthe grisea isolates on appressorium formation by cAMP and hydrophobicity of contact surface. Korean J. Plant Pathol. 10:254-260.

15. Lee, Y. H., and Dean, R. A. 1993. cAMP regulates infection structure formation inthe plant pathogenic fungus Magnaporthe grisea. Plant Cell 5:693-700

16. Mitchell, T. K., and Dean, R. A. 1995. The cAMP-dependent protein kinase catalytic subunit is required for appressorium formation and pathogenicity by the rice blast pathogen Magnaporthe grisea. Plant Cell 7:1869-1878.

17. Orlowski, M. 1995. Gene expression in Mucor dimorphism. Can. J. Bot. 73S:326-334.

18. Ou, S. H. 1985. Rice Diseases. 2nd ed. Commonwealth Mycological Institute, Kew, England.

19. Pegg, A. E., and Peter, P. M. 1982. Polyamine metabolism and function. Am. J. Physiol. 243:212-221.

20. Podila, G. K., Rogers, L. M., and Kolattukudy, P. E. 1993. Chemical signals from avocado surface wax trigger germination and appressorium formation in Colletotrichum gloeosporioides. Plant Physiol. 103:267-272.

21. Rajam, M. V., Weinstein, L. H., and Galston, A. W. 1985. Prevention of a plant disease by specific inhibition of fungal polyamine biosynthesis. Proc. Natl. Acad. Sci. USA 82:6874-6878.

22. Reitz, M., Walters, D., and Moerschbacher, B. 1995. Germination and appressorial formation by uredospores of Uromyces viciae-fabae exposed to inhibitors of polyamine. Eur. J. Plant Pathol. 101:573-578.

23. Reyna-Lopez, G., and Ruiz-Herrera, J. 1993. Polyamines and the phorogenesis of Mucorales. Exp. Mycol. 17:79-89.

24. Ruiz-Herrera, J. 1994. Polyamines, DNA methylation, and fungal differentiation. Crit. Rev. Microbiol. 20:143-150.

25. Ruiz-Herrera, J., and Calvo-Mendez, C. 1987. Effect of ornithine decarboxylase inhibitors on the germination of sporangiospores of Mucorales. Exp. Mycol. 11:287-296. 
26. Shapira, R., Altman, A., Henis, Y., and Chet, I. 1989. Polyamines and ornithine decarboxylase activity during growth and differentiation in Sclerotium rolfsii. J. Gen. Microbiol. 135:1361-1367.

27. Smith, T. A., Barker, J. H. A., and Owen, W. J. 1992. Insensitivity of Septoria tritici and Ustilago maydis to inhibitors of ornithine decarboxylase. Mycol. Res. 96:395-400.

28. Tabor, C. W., and Tabor, H. 1985. Polyamines in microorganisms. Microbiol. Rev. 49:81-99.

29. Walters, D. R. 1995. Inhibition of polyamine biosynthesis in fungi. Mycol. Res. 99:129-139.
30. Walters, D. R., Keenan, J. P., Cowley, T., McPherson, A., and Havis, N. D. 1995. Inhibition of polyamine biosynthesis in Phytophthora infestans and Pythium ultimum. Plant Pathol. 44:80-85.

31. Wright, J. M., and Boyle, S. M. 1982. Negative control of ornithine decarboxylase and arginine decarboxylase by adenosine- $3^{\prime}: 5^{\prime}$-cyclic monophosphate in Escherichia coli. Mol. Gen. Genet. 186:482-487.

32. Xiao, J. Z., Watanabe, T., Kamakura, T., Ohshima, A., and Yamakuchi, I. 1994. Studies on cellular differentiation of Magnaporthe grisea. Physicochemical aspects of substratum surfaces in relation to appressorium formation. Physiol. Mol. Plant Pathol. 44:227-236. 